\title{
OBSERVAÇÃO ADAPTATIVA NEURAL COM CONVERGÊNCIA ASSINTÓTICA NA PRESENÇA DE PARÂMETROS VARIANTES NO TEMPO E DISTÚRBIOS
}

\author{
José A. Ruiz Vargas* \\ vargas@ieee.org
}

\author{
Elder M. Hemerly* \\ hemerlyaita.br
}

* Departamento Sistemas e Controle, Instituto Tecnológico de Aeronáutica

12228-900 São José dos Campos, São Paulo, Brasil

\begin{abstract}
In this paper a scheme based on neural networks for adaptive observation of a class of uncertain continuous nonlinear systems in the presence of time-varying parameters and non-vanishing disturbances is proposed. Using standard Lyapunov procedures and an adaptive bounding technique, the state error convergence to zero is proved, even when approximation error and disturbances are present, while guaranteeing uniform ultimate boundedness of all others estimation errors (weight, parameter and bounding function). A simulation example to illustrate the application and performance of the proposed algorithm is provided.
\end{abstract}

KEYWORDS: Adaptive observers, nonlinear systems, neural networks, Lyapunov methods.

\section{RESUMO}

Neste artigo é proposto um esquema baseado em redes neurais para estimação adaptativa dos estados de uma classe de sistemas não-lineares contínuos, incertos, variantes no tempo e sujeita a distúrbios. Prova-se, via argumentos usuais de Lyapunov e uma técnica de limitação adaptativa (adaptive bounding technique), que o erro residual de observação converge para zero, inclusive na presença de erros de aproxi-

\footnotetext{
Artigo submetido em 21/09/2005

1a. Revisão em 19/08/2006

2a. Revisão em 24/01/2007

3a. Revisão em 06/11/2007

Aceito sob recomendação do Editor Associado Prof. Ivan Nunes da Silva
}

mação e distúrbios, enquanto os erros de estimação (pesos, parâmetros e função limitadora) permanecem limitados. Um exemplo de simulação é apresentado para ilustrar a aplicação e desempenho do esquema proposto.

PALAVRAS-CHAVE: Observadores adaptativos, sistemas não-lineares, redes neurais artificiais, métodos de Lyapunov

\section{INTRODUÇÃO}

Observadores são estimadores de estado para sistemas determinísticos, isto é, sistemas nos quais os ruídos de processo e de medidas não são significativos. Introduzidos por Luenberger (1966), os observadores de estado possuem diversas aplicações práticas, tais como monitoramento, controle e detecção de falhas. São empregados para obter uma estimativa $\hat{x}$ do vetor de estado verdadeiro $x$ a partir das saídas disponíveis, em situações usuais nas quais o número de sensores $(q)$ é menor que a dimensão do vetor de estado $(n)$. Tipicamente se tem $q<n$, devido à dificuldade de instalação ou elevado custo do sensor. Assim, pode-se entender o observador como sendo um software que substitui um componente de hardware. Deste modo, o sucesso desta substituição depende basicamente da qualidade do modelo do sistema. Portanto, o uso de observador é justificado para classes de sistemas para os quais é possível uma modelagem acurada o suficiente. Caso contrário, faz-se necessário utilizar um observador adaptativo, no qual as tarefas de reconstrução do estado e de modelagem são executadas simultaneamente.

Há na literatura pelo menos três metodologias para projeto 
de observadores adaptativos. A primeira é baseada em uma transformação de estado não-linear, de forma a se obter uma equação de erro linear, que possibilita que técnicas lineares possam ser empregadas (Marino \& Tomei, 1995; Bastin \& Gevers, 1988). Por exemplo, em Marino \& Tomei, (1995), foram estabelecidas as condições para a existência desta transformação. A segunda metodologia emprega o sistema original, mas sujeito a hipóteses convenientes, na seleção da estrutura do observador e obtenção de equação de erro (Rajamani, 1995; Cho \& Rajamani, 1997; Zhu \& Han, 2002). O terceiro método de projeto é baseado em aproximadores on-line, como por exemplo RNAs e sistemas nebulosos, para parametrização do sistema. Como exemplos desta abordagem podem ser citados Zhu et alii, (1997), Kim et alii, (1997), Vargas \& Hemerly, (1999), Choi \& Farrel, (2001). Convém ressaltar que as duas primeiras metodologias requerem o conhecimento prévio da estrutura do sistema, enquanto que a terceira não, uma vez que os aproximadores on-line têm estrutura conhecida a priori.

Em particular, as principais características que fundamentam o emprego de RNAs em observação adaptativa incluem: 1) RNAs têm estrutura conhecida, 2) RNAs podem aproximar sistemas não-lineares complexos via aprendizagem, sempre que satisfeitas as hipóteses de aproximação universal e regularidade (Polycarpou \& Ioannou, 1991), e 3) RNAs são adequadas ao processamento paralelo e exibem tolerância a falhas. Portanto, mediante o emprego de RNAs o problema de determinação de estrutura é simplificado, sendo o observador escolhido com base na parametrização neural, onde seus pesos são ajustados com leis de adaptação projetadas usualmente com base na análise de estabilidade. Como exemplos podem ser citados Zhu et alii, (1997), Kim et alii, (1997), Vargas \& Hemerly, (1999), Choi \& Farrel, (2001), onde foi considerada a observação adaptativa de varias classes de sistemas incertos contínuos com parâmetros constantes e desconhecidos, sendo os pesos dos observadores neurais empregados ajustados com leis de adaptação projetadas com base na análise de estabilidade. Em todos estes trabalhos, contudo, unicamente foi provado que os erros de observação residuais são limitados e, em geral, de magnitude proporcional a limitantes superiores dos erros de aproximação e distúrbios. A não garantia de convergência assintótica na presença de distúrbios não é um problema exclusivo da observação neural. Trabalhos recentes em observação não-linear baseada no conhecimento prévio da dinâmica dominante do sistema, vide Zhang, (2002), Marino, et alii, (2001), Wang \& Gao, (2003), e suas referências, também apresentam esta peculiaridade.

Por exemplo, em Marino et alii, (2001), foi proposto um observador adaptativo para uma classe de sistemas não-lineares multiple-input single output (MISO), cuja estrutura assumese conhecida a priori. Embora as hipóteses restritivas de conhecimento prévio da estrutura e saída escalar facilitem o projeto do observador, somente foi provado que o erro de observação converge para zero sob a hipótese crucial de inexistência ou convergência assintótica para zero dos distúrbios. Convém ressaltar que observadores adaptativos contínuos em malha aberta para sistemas não-lineares incertos muliple-input multipl-output (MIMO), que assegurem convergência assintótica do erro de observação para zero, inclusive na presença de distúrbios limitados, até onde os autores conhecem, são inexistentes na literatura.

Motivado pelos fatos anteriores, neste artigo é proposto um esquema, baseado em RNAs, para observação assintótica de uma classe de sistemas não-lineares MIMO, nos quais os parâmetros e distúrbios são limitados, desconhecidos e variantes no tempo. Mais precisamente, o observador proposto assegura, ao contrário dos propostos em Zhu et alii, (1997), Kim et alii, (1997), Vargas \& Hemerly, (1999), Choi \& Farrel, (2001), Zhang, (2002), Marino et alii, (2001), e Wang \& Gao, (2003), que o erro de observação converge assintóticamente para zero, inclusive na presença de erros de aproximação e distúrbios limitados. Para tanto, o sistema incerto foi parametrizado via RNAs lineares nos pesos, para contornar o empecilho do sistema incerto ter estrutura maiormente desconhecida. Desta forma o sistema parametrizado forneceu parte da estrutura do observador. Adicionalmente, um ganho de realimentação dinâmico foi também introduzido no observador para melhoria de desempenho, ao contrário do observador de Luenberger que emprega um ganho de realimentação constante (Luenberger, 1966). Com base na análise de estabilidade e usando uma técnica de limitação adaptativa (Polycarpou, 1996), mecanismos de adaptação são projetados para os parâmetros, pesos e ganho de realimentação, objetivando garantir que o observador seja assintoticamente estável, isto é, a convergência do erro de observação para zero. O observador proposto estende os resultados prévios em Rajamani, (1995), Cho \& Rajamani, (1997), Zhu \& Han, (2002), Zhu et alii, (1997), Kim et alii, (1997), Vargas \& Hemerly, (1999), Choi \& Farrel, (2001), Zhang, (2002), Marino et alii, (2001), e Wang \& Gao, (2003), uma vez que tem aplicação mais ampla, pois não requer o conhecimento prévio das não-linearidades do sistema, e assegura convergência assintótica do erro de observação para zero, inclusive na presença de distúrbios.

A principal contribuição deste trabalho é a proposição de uma metodologia, para a estimação do estado de uma classe de sistemas incertos MIMO, que assegura a convergência assintótica do erro de observação, apesar da presença de parâmetros variantes no tempo, erros de aproximação e distúrbios. Adicionalmente, uma vez que a metodologia de projeto proposta também pode ser usada sem RNAs, por exemplo quando as não-linearidades dominantes têm estrutura conhecida a priori, como em Marino \& Tomei, (1995), Bastin \& Gevers, (1988), Rajamani, (1995), Cho \& Rajamani, (1997), 
Zhu \& Han, (2002), Zhang, (2002), Marino, et alii, (2001), e Wang \& Gao, (2003), o presente trabalho estende o estado da arte em observação adaptativa baseada em RNAs e, em particular, em observação não-linear.

O restante do trabalho é organizado conforme a seguir. $\mathrm{Na}$ Seção 2 são apresentadas as classes de RNAs utilizadas na parametrização das não-linearidades desconhecidas. Na Seção 3 o problema de observação adaptativa é formulado. Já na Seção 4 o modelo para identificação e os erros associados são apresentados. Na Seção 5 é apresentado o principal resultado do artigo, a prova de estabilidade assintótica do erro de observação. Um exemplo de simulação é discutido na Seção 6. Finalmente, na Seção 7, as principais contribuições do trabalho são resumidas.

\section{REDES NEURAIS ARTIFICIAIS PARA- METRIZÁVEIS LINEARMENTE}

As redes neurais parametrizáveis linearmente (RNAPLs) podem ser expressas matematicamente como

$$
\rho_{n n}(W, \zeta)=W \pi(\zeta)
$$

onde $W \in \Re^{n \times L_{\rho}}, \zeta \in \Re^{L_{\zeta}}$ e $\pi: \Re^{L_{\zeta}} \mapsto \Re^{L_{\rho}}$ é um vetor de funções básicas que pode ser considerado como uma função vetorial não-linear, cujos argumentos são pré-processados por uma função escalar $s(\cdot)$, e $n, L_{\rho}, L_{\zeta}$ são inteiros estritamente positivos. As funções escalares $s(\cdot)$ usualmente utilizadas incluem sigmoide, tanh, gaussiana, Hardy's, inverse Hardy's multiquadratic, etc (Ge et alli, 2002). Entretanto, neste artigo considera-se somente a classe de RNAPLs nas quais $s(\cdot)$ é limitado, uma vez que neste caso tem-se,

$$
\|\pi(\zeta)\| \leq \pi_{0}
$$

sendo $\pi_{0}$ uma constante estritamente positiva.

A classe de RNAPLs consideradas neste trabalho inclui radial basis function neural networks (RBFNNs) (Sanner \& Slotine, 1992), wavelet networks (Zhang \& Benveniste, 1992), high order neural nertworks (HONNs) (Kosmatopoulos et alli, 1995), e também outros aproximadores parametrizáveis linearmente como Takagi-Sugeno fuzzy systems (Wang, 1994), os quais satisfazem a propriedade de aproximação universal e regularidade (Polycarpou \& Ioannou, 1991):

Propriedade 1: Para quaisquer constante $\varepsilon_{0}>0$ e função $f \in C[\Omega]$ existe uma matriz $W^{*}=W$, onde $W^{*}$ é uma matriz "ótima" e $L_{\rho}$ é suficientemente grande, tal que,

$$
\sup _{\zeta \in \Omega}\left|f(\zeta)-W^{*} \pi(\zeta)\right| \leq \varepsilon_{0}
$$

onde $C[\Omega]$ denota o espaço de funções contínuas definidas em um domínio compacto $\Omega \subset \Re^{n},|\cdot|$ denota o valor absoluto se o argumento é um escalar. Se o argumento é uma função vetorial em $\Re^{n}$ então $|\cdot|$ denota qualquer norma em $\Re^{n}$.

Propriedade 2: A saída da RNA é continua com respeito aos seus argumentos e satisfaz a condição de Lipschitz localmente para todo $(\zeta, W)$ finito.

\section{FORMULAÇÃO DO PROBLEMA}

Considere a classe de sistemas não-lineares representada por $\dot{x}=A x+B\left[f(x, u)+\sum_{i=1}^{p} g_{i}(x, u) \theta_{i}(t)+h(x, u, \theta, t)\right]$

$$
y=C x
$$

onde $x \in X$ é o vetor de estados de dimensão $n, u \in U$ é um vetor de entradas admissíveis de dimensão $m, y \in Y$ é o vetor de saídas de dimensão $q, \theta \in V$ é um vetor de parâmetros (variante no tempo e incerto) de dimensão $p, f: X \times U \mapsto$ $\Re^{r}$ e $g_{i}: X \times U \mapsto \Re^{r}, \quad \forall i=1,2, . ., p$ são mapeamentos contínuos desconhecidos, $h: X \times U \times V \times[0, \infty) \mapsto \Re^{r}$ é um vetor de distúrbios desconhecido, $A, B$ e $C$ são matrizes conhecidas de dimensões apropriadas. Com a finalidade de ter o problema bem colocado, assuma que $X, U, V$ são conjuntos compactos e que $f, g_{i}$ e $h$ são Lipschitz localmente com respeito a $x$ em $X \times U \times V \times[0, \infty)$, tal que (4) tem uma única solução passando por $x(0)$.

Assume-se que as seguintes hipóteses possam ser estabelecidas

Hipótese 1: Na região $X \times U \times V \times[0, \infty)$

$$
\|h(x, u, \theta, t)\| \leq h_{0}
$$

onde $\|\cdot\|$ é a norma Euclideana e $h_{0}$ é uma constante positiva.

Hipótese 2: $\mathrm{O}$ parâmetro $\theta(t)=$ $\left[\begin{array}{llll}\theta_{1}(t) & \theta_{2}(t) & \cdots & \theta_{p}(t)\end{array}\right]^{T}$ é tal que $\theta \in C^{1}$, isto é, $\theta$ possui primeira derivada temporal continua, $\theta_{i}(t)$ têm sinal constante conhecido e $d \theta_{i}^{2}(t) / d t \leq 0 \quad \forall i=1,2, . ., p$. 
Hipótese 3: Existe uma matriz simétrica positiva definida $P \in \Re^{n \times n}$ e uma matriz de ganho $L \in \Re^{n \times q}$ tais que

$$
\begin{gathered}
P(A-L C)+(A-L C)^{T} P+\left(\rho_{1}+\rho_{2}\right) P P / 2 \\
+\left(\rho_{1}+\rho_{2}\right) I / 2=-Q<0 \\
B^{T} P=C^{*}
\end{gathered}
$$

onde $\rho_{1}$ e $\rho_{2}$ são constantes estritamente positivas, $Q$ é uma matriz positiva definida e cada linha de $C^{*}$ está no span das linhas de $C$.

Comentário 1: A Hipótese 1, a qual impõe um limitante superior sobre a norma dos distúrbios, é completamente usual em identificação. A Hipótese 2 impõe que a “energia' de $\theta$ seja constante ou continuamente dissipada, o qual implica

$$
\|\theta(t)\| \leq \beta_{0}
$$

para alguma constante positiva $\beta_{0}$.

Comentário 2: A Hipótese 3 impõe que o par $(A, C)$ seja detectável e a parte linear do sistema dissipativa (strictly positive real (Ioannou \& Sun, 1996)). Em Rajamani, (1998), são fornecidas condições necessárias e suficientes sobre $L$ para assegurar que (7) seja verificada: $L$ é escolhida tal que $(A-L C)$ é estável e

$$
\min _{\omega \in \Re^{+}} \sigma(A-L C-j \omega I)>\left(\rho_{1}+\rho_{2}\right) / 2
$$

onde $\sigma(\cdot)$ denota o valor singular mínimo de uma matriz e $j=\sqrt{-1}$.

Objetiva-se projetar um observador adaptativo para (4), baseado em RNAPLs, o qual assegure a convergência assintótica do erro residual de observação para zero, apesar da presença de erros de aproximação e distúrbios limitados.

\section{MODELO PARA IDENTIFICAÇÃO E EQUAÇÃO DO ERRO DE OBSERVA- ÇÃO}

Nesta seção é proposta a estrutura do observador e, com base na mesma, é obtida a equação do erro de observação associada com o problema.

Com base em (3), usando-se RNAPLs, os mapeamentos $f(x, u)$ e $g_{i}(x, u)$ podem ser substituídos por $W_{f}^{*} \pi_{f}(x, u)$ e $W_{g i}^{*} \pi_{g i}(x, u)$ mais os erros de aproximação $\varepsilon_{f}(x, u)$ e $\varepsilon_{g i}(x, u)$. Mais exatamente, (4) pode ser escrita como

$$
\begin{aligned}
\dot{x} & =A x+B W_{f}^{*} \pi_{f}(x, u)+B \sum_{i=1}^{p} W_{g i}^{*} \pi_{g i}(x, u) \theta_{i} \\
& +B \varepsilon_{f}(x, u)+B \sum_{i=1}^{p} \varepsilon_{g i}(x, u) \theta_{i}+B h(x, u, \theta, t)
\end{aligned}
$$

onde $W_{f}^{*} \in \Re^{r x L_{f}}$ e $W_{g i}^{*} \in \Re^{r x L_{g i}}$ são matrizes "ótimas", requeridas somente para propósitos analíticos, que podem ser definidas como

$$
\begin{gathered}
W_{f}^{*}:=\underset{\hat{W}_{f} \in \Gamma_{f}}{\arg \min }\left\{\sup _{\substack{x \in X, u \in U}}\left|f(x, u)-\hat{W}_{f} \pi_{f}(x, u)\right|\right\}, \\
W_{g i}^{*}:=\underset{\hat{W}_{g i} \in \Gamma_{g i}}{\arg \min }\left\{\sup _{\substack{x \in X, u \in U}}\left|g_{i}(x, u)-\hat{W}_{g i} \pi_{g i}(x, u)\right|\right\}
\end{gathered}
$$

$\operatorname{com} \Gamma_{f}=\left\{\hat{W}_{f} \mid\left\|\hat{W}_{f}\right\|_{F} \leq \alpha_{\hat{W}_{f}}\right\}, \quad \Gamma_{g i}=$ $\left\{\hat{W}_{g i} \mid\left\|\hat{W}_{g i}\right\|_{F} \leq \alpha_{\hat{W}_{g i}}\right\}$, $\|\cdot\|_{F}$ é a norma de Frobenius, $\alpha_{\hat{W}_{f}}$ e $\alpha_{\hat{W}_{g i}}$ são constantes estritamente positivas, as quais dependem das leis de ajuste dos pesos, $\hat{W}_{f}$ e $\hat{W}_{g i}$ são estimações de $W_{f}^{*}$ e $W_{g i}^{*}$, respectivamente. Os erros de aproximação $\varepsilon_{f}(x, u)$ e $\varepsilon_{g i}(x, u)$, correspondentes a $W_{f}^{*} \mathrm{e}$ $W_{g i}^{*}$, podem ser definidos como

$$
\begin{aligned}
& \varepsilon_{f}(x, u):=f(x, u)-W_{f}^{*} \pi_{f}(x, u), \\
& \varepsilon_{g i}(x, u):=g_{i}(x, u)-W_{g i}^{*} \pi_{g i}(x, u)
\end{aligned}
$$

Uma vez que $X, U$ são conjuntos compactos e usando-se (2), na região $X \times U$ tem-se

$$
\left\|\varepsilon_{f}(x, u)\right\| \leq \varepsilon_{f 0}, \quad\left\|\varepsilon_{g i}(x, u)\right\| \leq \varepsilon_{g i 0}
$$

onde $\varepsilon_{f 0}$ e $\varepsilon_{g i 0}$ são constantes positivas. 
Comentário 3: É importante ressaltar que $W_{f}^{*}$ e $\varepsilon_{f}(x, u)$ $\left(W_{g i}^{*}\right.$ e $\left.\varepsilon_{g i}(x, u)\right)$ podem não ser únicos. Entretanto, $\left\|\varepsilon_{f}(x, u)\right\|\left(\left\|\varepsilon_{g i}(x, u)\right\|\right)$ é único por (12).

A estrutura (11) sugere um observador da forma

$$
\begin{aligned}
& \dot{\hat{x}}=A \hat{x}+ \\
& B\left[\hat{W}_{f} \pi_{f}(\hat{x}, u)+\sum_{i=1}^{p} \hat{W}_{g i} \pi_{g i}(\hat{x}, u) \hat{\theta}_{i}-l\left(C^{*} \tilde{x}, \hat{\psi}, t\right)\right] \\
& -L \tilde{y}
\end{aligned}
$$

onde $\hat{x}$ é o estado estimado, $\tilde{x}=\hat{x}-x$ é o erro de observação, $\hat{\theta}$ é o vetor de parâmetros estimado, $\tilde{y}=C \hat{x}-y$ é o erro de estimação da saída, $\hat{\psi}$ e $l$ são, respectivamente, uma função escalar (limitadora) e vetorial, as quais serão definidas apropriadamente na próxima seção (vide (28) e (24)). Mostrar-seá na próxima seção que o observador (15), com mecanismos de adaptação adequadamente projetados para $\hat{W}_{f}, \hat{W}_{g i}, \hat{\theta}, \hat{\psi}$ e $l$, assegura a convergência do erro de observação para zero, inclusive na presença de erros de aproximação e distúrbios.

Comentário 4: É importante ressaltar que o termo $C^{*} \tilde{x}$ em (15), onde $\tilde{x}$ não é disponível para medições, pode ser determinado via $\tilde{y}$, uma vez que $C^{*}$ está no espaço gerado pelas linhas de $C$ e, portanto, existe uma matriz $T$ tal que $C^{*}=T C$. Objetivando-se determinar $T$, note que a matriz $C$ pode ser decomposta em valores singulares como

$$
C=U \sum V^{T}
$$

donde a inversa generalizada ou pseudo-inversa de $C$ resulta,

$$
C^{+}=V \sum^{+} U^{T}
$$

onde $U \in \Re^{q \times q}$ e $V \in \Re^{n \times n}$ são matrizes ortogonais,

$$
\begin{gathered}
\sum=\left[\begin{array}{cc}
D & 0_{k \times(n-k)} \\
0_{(q-k) \times k} & 0_{(q-k) \times(n-k)}
\end{array}\right], \\
D=\operatorname{diag}\left(\sigma_{i}, i=1,2, . ., k\right), \sigma_{i}>0
\end{gathered}
$$

são os valores singulares de $C, k$ é o posto de $C$ e

$$
\sum^{+}=\left[\begin{array}{cc}
D^{-1} & 0_{k \times(q-k)} \\
0_{(n-k) \times k} & 0_{(n-k) \times(q-k)}
\end{array}\right]
$$

(Noble \& Daniel, 1977). Então, $T=C^{*} C^{+}$, pois esta matriz satisfaz a equação $C^{*}=T C$, e conseqüentemente $C^{*} \tilde{x}=$ $T C \tilde{x}=T \tilde{y}$.
Com base em (11) e (15), obtém-se a equação do erro de observação

$$
\begin{gathered}
\dot{\tilde{x}}=(A-L C) \tilde{x}+B\left[\tilde{W}_{f} \pi_{f}(\hat{x}, u)+\right. \\
\sum_{i=1}^{p} \tilde{W}_{g i} \pi_{g i}(\hat{x}, u) \hat{\theta}_{i}+\sum_{i=1}^{p} W_{g i}^{*} \pi_{g i}(\hat{x}, u) \tilde{\theta}_{i}+ \\
\left.d(\hat{x}, x, u, \theta, t)-l\left(C^{*} \tilde{x}, \hat{\psi}, t\right)\right] \quad(18) \\
\text { onde } \tilde{W}_{f}=\hat{W}_{f}-W_{f}^{*}, \tilde{W}_{g i}=\hat{W}_{g i}-W_{g i}^{*}, \tilde{\theta}_{i}=\hat{\theta}_{i}-\theta_{i} \mathrm{e} \\
d(\hat{x}, x, u, \theta, t)=W_{f}^{*}\left[\pi_{f}(\hat{x}, u)-\pi_{f}(x, u)\right] \\
\quad+\sum_{i=1}^{p} W_{g i}^{*}\left[\pi_{g i}(\hat{x}, u)-\pi_{g i}(x, u)\right] \theta_{i}-\varepsilon_{f}(x, u) \\
-\sum_{i=1}^{p} \varepsilon_{g i}(x, u) \theta_{i}-h(x, u, \theta, t)
\end{gathered}
$$

\section{LEIS DE ADAPTAÇÃO PARA OS PESOS E ANÁLISE DE ESTABILIDADE}

Nesta seção, objetivando-se assegurar a convergência do erro de predição, são projetadas as leis de adaptação para os pesos, parâmetros e função de realimentação $l$ em (15). Para tanto, empregam-se argumentos usuais de Lyapunov e uma técnica de limitação adaptativa (Polycarpou, 1996), para assegurar estabilidade assintótica do erro de observação e limitação dos erros de estimação dos pesos, parâmetros e função limitadora. Basicamente, a técnica de limitação adaptativa permite assegurar que o limitante superior de $\dot{V}$, a derivada em relação ao tempo de uma candidata a função de Lyapunov $V$, seja zero. Isto é forçado pela introdução de um sinal auxiliar ou função limitadora no termo de realimentação dinâmico $l$ do observador. A função limitadora é projetada com base na análise de estabilidade.

Inicialmente, objetivando-se provar que os erros estimação são ultimamente limitados (Ioannou \& Sun, 1996), considere o vetor composto de erros

$$
\begin{gathered}
\tilde{\chi}=\left[\tilde{x}^{T} \operatorname{vec}\left(\tilde{W}_{f}\right)^{T} \operatorname{vec}\left(\tilde{W}_{g 1}\right)^{t} \ldots\right. \\
\left.\cdots \operatorname{vec}\left(\tilde{W}_{g p}\right)^{T} \tilde{\psi} \tilde{\theta}^{T}\right]^{T}
\end{gathered}
$$

e a maior bola na região de operação

$$
B_{R}=\{\tilde{\chi} \mid\|\tilde{\chi}\| \leq R\}
$$

de forma que para todo $\tilde{\chi} \in B_{R}$ tem-se que $(x, u) \in X \times U$, 
onde $\tilde{\psi}=\hat{\psi}-\psi^{*}$, sendo $\psi^{*}$ e $R$ constantes estritamente positivas.

Hipótese 4: Assuma que

$$
R>\gamma_{0} \sqrt{\frac{T_{M}}{T_{m}}} \geq \gamma_{0}
$$

onde

$$
\begin{gathered}
T_{M}=\frac{1}{2} \max \left[2 \lambda_{\max }(P), \alpha_{f}^{-1}, \alpha_{g i}^{-1}, \alpha_{\psi}^{-1}, \alpha_{\theta}^{-1},\right. \\
i=1,2, \cdots, p] \\
T_{m}=\frac{1}{2} \min \left[2 \lambda_{\min }(P), \alpha_{f}^{-1}, \alpha_{g i}^{-1}, \alpha_{\psi}^{-1}, \alpha_{\theta}^{-1},\right. \\
i=1,2, . ., p] \\
\gamma_{0}=\max \left(\frac{\psi_{1}^{2}\left\|C^{*}\right\|}{4 \alpha_{1} \psi_{0}}, \frac{\psi_{1}}{2 \sqrt{\psi_{0} c_{f \min }}}, \frac{\psi_{1}}{2 \sqrt{\psi_{0} c_{g i \min }}},\right. \\
\left.\frac{\psi_{1}}{2 \psi_{0}}, \frac{\psi_{1}}{2 \sqrt{\psi_{0} c_{\theta \min }}}, i=1,2, . ., p\right)
\end{gathered}
$$

$\lambda_{\min }(\Gamma)\left(\lambda_{\max }(\Gamma)\right)$ é o menor (maior) autovalor de uma matriz quadrada $\Gamma, \alpha_{f}, \alpha_{g i}, \alpha_{\psi}, \alpha_{\theta}, \psi_{0}, \psi_{1}$ são constantes estritamente positivas, $\alpha_{1}=\lambda_{\min }(Q), c_{f \min }=\lambda_{\min }\left(C_{f}\right)$, $C_{f}=\operatorname{diag}\left(c_{f j}, j=1,2, . ., n\right), c_{g i \min }=\lambda_{\min }\left(C_{g i}\right)$, $C_{g i}=\operatorname{diag}\left(c_{g i j}, j=1,2, . ., n\right), c_{g i j}>0, c_{\theta \min }=$ $\lambda_{\min }\left(C_{\theta}\right), C_{\theta}=\operatorname{diag}\left(c_{\theta}, i=1,2, . ., p\right)$ e $c_{\theta}{ }_{i}>0$.

Considere também a seguinte função positiva definida (função de Lyapunov candidata (Ioannou \& Sun, 1996))

$$
\begin{aligned}
& V=\tilde{x}^{T} P \tilde{x}+\operatorname{tr}\left(\tilde{W}_{f}^{T} \alpha_{f}^{-1} \tilde{W}_{f}\right) / 2+ \\
& \sum_{i=1}^{p} \operatorname{tr}\left(\tilde{W}_{g i}^{T} \alpha_{g i}^{-1} \tilde{W}_{g i}\right) / 2+\tilde{\psi} \alpha_{\psi}^{-1} \tilde{\psi} / 2+\sum_{i=1}^{p} \tilde{\theta}_{i} \alpha_{\theta}^{-1} \tilde{\theta}_{i} / 2
\end{aligned}
$$

onde $\operatorname{tr}(\cdot)$ é o operador traço, e defina o máximo e mínimo valores de $V$ sobre os contornos de $B_{\gamma 0}=\left\{\tilde{\chi} \mid\|\tilde{\chi}\| \leq \gamma_{0}\right\}$ e $B_{R}$, respectivamente, como $V_{M}=\max \|\tilde{\chi}\|=\gamma_{0} V=\gamma_{0}^{2} T_{M}$

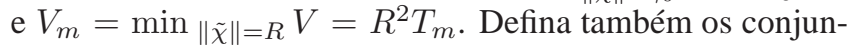
$\operatorname{tos} \Omega_{M}=\left\{\tilde{\chi} \mid V \leq V_{M}\right\} \mathrm{e}$

$$
\Omega_{m}=\left\{\tilde{\chi} \mid V \leq V_{m}\right\}
$$

Comentário 5: Uma vez que $V_{M}<V_{m}$ pela Hipótese 4, conclui-se que $\Omega_{M} \subset \Omega_{m}$.

O resultado principal do trabalho é estabelecido e provado na seqüência.

Teorema 1: Considere a classe de sistemas não-lineares incertos descrito por (4), satisfazendo as Hipótese 1-4, o observador (15) com

$$
\begin{gathered}
l\left(C^{*} \tilde{x}, \hat{\psi}, t\right)=\left[\frac{\psi_{1} \hat{\psi}}{\left\|C^{*} \tilde{x}\right\|+z(t)}\right] C^{*} \tilde{x} \\
z(t)=z_{0} \exp \left(-z_{1} t\right)
\end{gathered}
$$

e as leis adaptativas,

$$
\dot{\hat{W}}_{f}=-2 \alpha_{f}\left[C_{f}\left\|C^{*} \tilde{x}\right\|\left(\hat{W}_{f}-W_{f 0}\right)+C^{*} \tilde{x} \pi_{f}^{T}(\hat{x}, u)\right]
$$

$$
\begin{aligned}
& \dot{\hat{W}}_{g i}=-2 \alpha_{g i}\left[C_{g i}\left\|C^{*} \tilde{x}\right\|\left(\hat{W}_{g i}-W_{g i 0}\right)+\right. \\
& \left.+C^{*} \tilde{x} \pi_{g i}^{T}(\hat{x}, u) \hat{\theta}_{i}\right] \\
& \dot{\hat{\psi}}=-\alpha_{\psi}\left(2 \psi_{0}\left\|C^{*} \tilde{x}\right\| \hat{\psi}-\psi_{1}\left\|C^{*} \tilde{x}\right\|\right)
\end{aligned}
$$

$$
\dot{\hat{\theta}}_{i}=-\alpha_{\theta}\left[2 c_{\theta i}\left\|C^{*} \tilde{x}\right\| \hat{\theta}_{i}+\operatorname{sgn}\left(\theta_{i}\right)\left\|C^{*} \tilde{x}\right\|\right]
$$

Se os erros iniciais pertencem ao conjunto (23) e

$$
\hat{\psi}(0)>0
$$

$$
\hat{\theta}_{i}(0)\left\{\begin{array}{l}
>0 \text { se } \operatorname{sgn}\left(\theta_{i}\right)=-1 \\
<0 \text { se } \operatorname{sgn}\left(\theta_{i}\right)=1
\end{array}\right.
$$

$$
\psi_{1} \geq 2 \sqrt{\alpha_{0} \psi_{0}}
$$

onde 


$$
\begin{aligned}
& i=1,2, . ., p, \\
& z_{0}, z_{1}>0, \\
& W_{f 0} \in \Re^{r \times L_{f}}, \\
& W_{g i 0} \in \Re^{r \times L_{g i}} \text {, } \\
& \alpha_{0}=\alpha_{2}+\alpha_{3}+c_{f \max }\left\|W_{f}^{*}-W_{f 0}\right\|_{F}^{2}+ \\
& \sum_{i=1}^{p} c_{g i \max }\left\|W_{g i}^{*}-W_{g i 0}\right\|_{F}^{2}+\beta_{0}^{2} c_{\theta \min }+\left(\alpha_{\hat{\theta}}+\beta_{0}\right) \sqrt{p} \text {, } \\
& c_{f \max }=\lambda_{\max }\left(C_{f}\right), \\
& c_{g i \max }=\lambda_{\max }\left(C_{g i}\right) \text {, } \\
& \alpha_{2}=2\left(\alpha_{\hat{\theta}}+\beta_{0}\right)_{F}\left(\sum_{i=1}^{p} \pi_{g i 0}^{2}\left\|W_{g i}^{*}\right\|_{F}^{2}\right)^{\frac{1}{2}} \text {, } \\
& \pi_{g i 0}=\sup _{t \geq 0}\left\|\pi_{g i}(x(t), u(t))\right\| \text {, } \\
& \alpha_{3}=2\left[\varepsilon_{f 0}+\beta_{0}\left(\sum_{i=1}^{p} \varepsilon_{g i 0}^{2}\right)^{1 / 2}+h_{0}\right] \text {, } \\
& \alpha_{\hat{\theta}}=\max \left\{\frac{\sqrt{p}}{2 c_{\theta \min }},\|\hat{\theta}(0)\|\right\} \text {. }
\end{aligned}
$$

Então,

(i) $\tilde{\chi}(t)$ é ultimamente limitado.

(ii) $\lim _{t \rightarrow \infty} \tilde{x}(t)=0$.

Prova: Primeiramente mostra-se que o erro $\tilde{\theta}$ é limitado, independentemente dos outros sinais de erro, o qual permite que desigualdades necessárias para a prova de convergência sejam estabelecidas. Para tanto, considere a função positiva semi-definida (Lyapunov-like (Ioannou \& Sun, 1996))

$$
V_{\hat{\theta}}=\sum_{i=1}^{p} \hat{\theta}_{i} \alpha_{\theta}^{-1} \hat{\theta}_{i} / 2
$$

Derivando-se (34) em relação ao tempo e usando-se (29), obtém-se

$$
\begin{aligned}
\dot{V}_{\hat{\theta}}=-\left\|C^{*} \tilde{x}\right\| & {\left[\sum_{i=1}^{p} 2 c_{\theta} \hat{\theta}_{i}^{2}-\sum_{i=1}^{p}\left|\hat{\theta}_{i}\right|\right] } \\
& \leq-\left\|C^{*} \tilde{x}\right\|\|\hat{\theta}\|\left(2 c_{\theta \min }\|\hat{\theta}\|-\sqrt{p}\right)
\end{aligned}
$$

Portanto, a condição $\|\hat{\theta}\|>\frac{\sqrt{p}}{2 c_{\theta \min }}$ torna $\dot{V}_{\hat{\theta}} \leq 0$ fora do conjunto compacto $\Omega_{\hat{\theta}}=\left\{\hat{\theta} \mid\|\hat{\theta}\| \leq \alpha_{\hat{\theta}}\right\}$, onde $\alpha_{\hat{\theta}}=$ $\max \left\{\frac{\sqrt{p}}{2 c_{\theta \min }},\|\hat{\theta}(0)\|\right\}$, o qual assegura a limitação de $\hat{\theta}$. Uma vez que $\hat{\theta}(0) \in \Omega_{\hat{\theta}}$ pode ser concluído que

$$
\|\hat{\theta}(t)\| \leq \alpha_{\hat{\theta}}, \quad \forall t \geq 0
$$

Com base em (8), (36) e a Propriedade 2, podem ser estabelecidas as seguintes desigualdades

$$
\begin{aligned}
2 \tilde{x}^{T} P B \sum_{i=1}^{p} W_{g i}^{*} \pi_{g i}(\hat{x}, u) \tilde{\theta}_{i} \leq \alpha_{2} & \left\|C^{*} \tilde{x}\right\|+ \\
& +\rho_{1}\|P \tilde{x}\|\|\tilde{x}\|
\end{aligned}
$$

$$
2 \tilde{x}^{T} P B d \leq \alpha_{3}\left\|C^{*} \tilde{x}\right\|+\rho_{2}\|P \tilde{x}\|\|\tilde{x}\|
$$

onde $\rho_{1}=2\left(\alpha_{\hat{\theta}}+\beta_{0}\right)\|B\|_{F}\left(\sum_{i=1}^{p} \gamma_{g i}^{2}\left\|W_{g i}^{*}\right\|_{F}^{2}\right)^{\frac{1}{2}}$, $\rho_{2}=2\|B\|_{F}\left[\gamma_{f}\left\|W_{f}^{*}\right\|_{F}+\beta_{0}\left(\sum_{i=1}^{p} \gamma_{g i}^{2}\left\|W_{g i}^{*}\right\|_{F}^{2}\right)^{1 / 2}\right]$, sendo $\gamma_{f}$ e $\gamma_{g i}$ constantes de Lipschitz estritamente positivas.

Derivando-se agora (22) em relação ao tempo, obtém-se

$$
\begin{aligned}
\dot{V} & =\tilde{x}^{T} P \dot{\tilde{x}}+\dot{\tilde{x}}^{T} P \tilde{x}+\operatorname{tr}\left(\tilde{W}_{f}^{T} \alpha_{f}^{-1} \dot{\tilde{W}}_{f}\right) \\
& +\sum_{i=1}^{p} \operatorname{tr}\left(\tilde{W}_{g i}^{T} \alpha_{g i}^{-1} \dot{\tilde{W}}_{g i}\right)+\tilde{\psi} \alpha_{\psi}^{-1} \dot{\tilde{\psi}}+\sum_{i=1}^{p} \tilde{\theta}_{i} \alpha_{\theta}^{-1} \dot{\tilde{\theta}}_{i}
\end{aligned}
$$




$$
\begin{aligned}
& \dot{V}=-\tilde{x}^{T}\left[P(A-L C)+(A-L C)^{T} P\right] \tilde{x}+ \\
& +2 \tilde{x}^{T} P B \tilde{W}_{f} \pi_{f}(\hat{x}, u)+ \\
& +2 \tilde{x}^{T} P B \sum_{i=1}^{p} \tilde{W}_{g i} \pi_{g i}(\hat{x}, u) \hat{\theta}_{i}+ \\
& +2 \tilde{x}^{T} P B \sum_{i=1}^{p} W_{g i}^{*} \pi_{g i}(\hat{x}, u) \tilde{\theta}_{i}+ \\
& +2 \tilde{x}^{T} P B d-2 \tilde{x}^{T} P B l \\
& -2\left\|C^{*} \tilde{x}\right\| \operatorname{tr}\left[2 \tilde{W}_{f}^{T} C_{f}\left(\hat{W}_{f}-W_{f 0}\right)\right]+ \\
& -2 \operatorname{tr}\left[\tilde{W}_{f}^{T} C^{*} \tilde{x} \pi_{f}^{T}(\hat{x}, u)\right]+ \\
& -2\left\|C^{*} \tilde{x}\right\| \sum_{i=1}^{p} \operatorname{tr}\left[2 \tilde{W}_{g i}^{T} C_{g i}\left(\hat{W}_{g i}-W_{g i 0}\right)\right] \\
& -2 \sum_{i=1}^{p}\left[\tilde{W}_{g i}^{T} C^{*} \tilde{x} \pi_{g i}^{T}(\hat{x}, u) \hat{\theta}_{i}\right]+ \\
& -2 \psi_{0}\left\|C^{*} \tilde{x}\right\| \tilde{\psi} \hat{\psi}+\psi_{1} \tilde{\psi}\left\|C^{*} \tilde{x}\right\| \\
& -2\left\|C^{*} \tilde{x}\right\| \sum_{i=1}^{p} c_{\theta} \tilde{\theta}_{i} \hat{\theta}_{i}+ \\
& -\left\|C^{*} \tilde{x}\right\| \sum_{i=1}^{p} \operatorname{sgn}\left(\theta_{i}\right) \tilde{\theta}_{i}-\sum_{i=1}^{p} \tilde{\theta}_{i} \alpha_{\theta}^{-1} \dot{\theta}_{i}
\end{aligned}
$$

Lembrando agora que $\tilde{\theta}_{i}=\hat{\theta}_{i}-\theta_{i}$, pode ser estabelecido que

$$
\sum_{i=1}^{p} \tilde{\theta}_{i} \alpha_{\theta}^{-1} \dot{\theta}_{i} \geq 0
$$

já que $\sum_{i=1}^{p} \hat{\theta}_{i} \alpha_{\theta}^{-1} \dot{\theta}_{i} \geq 0$, devido à Hipótese 2 e condição (31), as quais asseguram que $\operatorname{sgn}\left(\hat{\theta}_{i}\right)=\operatorname{sgn}\left(\dot{\theta}_{i}\right), \forall i=$ $1,2, \ldots, p$, e $\sum_{i=1}^{p} \theta_{i} \alpha_{\theta}^{-1} \dot{\theta}_{i} \leq 0$ pela Hipótese 2.

Por outro lado, usando-se (8) e uma propriedade simples do traço resulta,

$$
\begin{aligned}
\operatorname{tr}\left[\tilde{W}_{f}^{T} C^{*} \tilde{x} \pi_{f}^{T}(\hat{x}, u)\right] & =\tilde{x}^{T} P B \tilde{W}_{f} \pi_{f}(\hat{x}, u) \\
\sum_{i=1}^{p}\left[\tilde{W}_{g i}^{T} C^{*} \tilde{x} \pi_{g i}^{T}(\hat{x}, u) \hat{\theta}_{i}\right] & =\sum_{i=1}^{p} \tilde{x}^{T} P B \tilde{W}_{g i} \pi_{g i}(\hat{x}, u) \hat{\theta}_{i}
\end{aligned}
$$

Também, vide Vargas \& Hemerly, (2005), para maiores detalhes, tem-se

$$
\begin{gathered}
2 \operatorname{tr}\left[\tilde{W}_{f}^{T} C_{f}\left(\hat{W}_{f}-W_{f 0}\right)\right] \\
\geq c_{f \min }\left\|\tilde{W}_{f}\right\|_{F}^{2}-c_{f \max }\left\|W_{f}^{*}-W_{f 0}\right\|_{F}^{2} \\
\sum_{i=1}^{p} \operatorname{tr}\left[2 \tilde{W}_{g i}^{T} C_{g i}\left(\hat{W}_{g i}-W_{g i 0}\right)\right] \\
\geq \sum_{i=1}^{p}\left[c_{g i \min }\left\|\tilde{W}_{g i}\right\|_{F}^{2}-c_{g i \max }\left\|W_{g i}^{*}-W_{g i 0}\right\|_{F}^{2}\right] \\
2 \tilde{\psi} \hat{\psi}=\tilde{\psi}^{2}+\hat{\psi}^{2}-\psi^{*^{2}} \\
\sum_{i=1}^{p} 2 c_{\theta i} \tilde{\theta}_{i} \hat{\theta}_{i}=\sum_{i=1}^{p} c_{\theta i}\left(\tilde{\theta}_{i}^{2}+\hat{\theta}_{i}^{2}-\theta_{i}^{2}\right)
\end{gathered}
$$

Logo, usando-se (37), (38), (41)-(47), $\|P \tilde{x}\|\|\tilde{x}\| \leq$ $\tilde{x}^{T}(P P+I) \tilde{x} / 2$ e ordenando termos, (40) implica

$$
\begin{gathered}
\dot{V} \leq-\tilde{x}^{T} Q \tilde{x}-2 \tilde{x}^{T} P B l-\left\|C^{*} \tilde{x}\right\|\left(c_{f \min }\left\|\tilde{W}_{f}\right\|_{F}^{2}\right. \\
+\sum_{i=1}^{p} c_{g i \min }\left\|\tilde{W}_{g i}\right\|_{F}^{2}+\psi_{0} \tilde{\psi}^{2}+c_{\theta \min }\|\tilde{\theta}\|^{2}-\psi_{0} \psi^{* 2} \\
\left.-\alpha_{0}-\psi_{1} \tilde{\psi}+\psi_{0} \hat{\psi}^{2}\right)
\end{gathered}
$$

Definindo-se agora $\psi^{*}=\frac{\psi_{1}+\sqrt{\psi_{1}^{2}-4 \alpha_{0} \psi_{0}}}{2 \psi_{0}},(48)$ reduz-se a

$$
\begin{array}{r}
\dot{V} \leq-\tilde{x}^{T} Q \tilde{x}-2 \tilde{x}^{T} P B l-\left\|C^{*} \tilde{x}\right\|\left(c_{f \min }\left\|\tilde{W}_{f}\right\|_{F}^{2}\right. \\
+\sum_{i=1}^{p} c_{g i \min }\left\|\tilde{W}_{g i}\right\|_{F}^{2}+\psi_{0} \tilde{\psi}^{2}+c_{\theta \min }\|\tilde{\theta}\|^{2}-\psi_{1} \hat{\psi} \\
\left.+\psi_{0} \hat{\psi}^{2}\right)
\end{array}
$$

Adicionalmente, completando-se o quadrado e uma vez que $\tilde{x}^{T} Q \tilde{x} \geq \alpha_{1}\left\|C^{*} \tilde{x}\right\|\|\tilde{x}\| /\left\|C^{*}\right\|_{F}$ e $\tilde{x}^{T}(t) P B l \geq 0, \forall t \geq$ 0 , decorre

$$
\begin{gathered}
\dot{V} \leq-\left\|C^{*} \tilde{x}\right\|\left[\alpha_{1}\|\tilde{x}\| /\left\|C^{*}\right\|_{F}+c_{f \min }\left\|\tilde{W}_{f}\right\|_{F}^{2}\right. \\
+\sum_{i=1}^{p} c_{g i \min }\left\|\tilde{W}_{g i}\right\|_{F}^{2}+\psi_{0} \tilde{\psi}^{2}+ \\
\left.c_{\theta \min }\|\tilde{\theta}\|^{2}-\psi_{1}^{2} /\left(4 \psi_{0}\right)\right]
\end{gathered}
$$


Defina o intervalo $[t, t+T]$ (o intervalo do tempo no qual a estimação é feita), como dois conjuntos disjuntos: $\Omega_{1}=$ $\left\{\left[t_{i}, t_{i+1}\right] \mid \tilde{x}(t)=0, \quad t \in\left[t_{i}, t_{i+1}\right]\right\}$ e $\Omega_{1}^{C}$. Para completar a prova de estabilidade considere os seguintes dois casos:

(i) Se $t \in \Omega_{1}$, com base em (50), tem-se que $\tilde{W}_{f}, \tilde{W}_{g i}, \tilde{\psi}$ e $\tilde{\theta}$ são limitados.

(ii) Se $t \in \Omega_{1}^{C}$, quaisquer uma das condições: $\|\tilde{x}\|>$ $\frac{\psi_{1}^{2}\left\|C^{*}\right\|_{F}}{4 \alpha_{1} \psi_{0}},\left\|\tilde{W}_{f}\right\|_{F}>\frac{\psi_{1}}{2 \sqrt{\psi_{0} c_{f \min }}},\left\|\tilde{W}_{g i}\right\|_{F}>\frac{\psi_{1}}{2 \sqrt{\psi_{0} c_{g i \min }}}$, $|\tilde{\psi}|>\frac{\psi_{1}}{2 \psi_{0}}$ ou $\|\tilde{\theta}\|>\frac{\psi_{1}}{2 \sqrt{\psi_{0} c_{\theta \text { min }}}}$, tornam $\dot{V}<0$ fora do conjunto $B_{\gamma_{0}}=\left\{\tilde{\chi} \in B_{R} \mid\|\tilde{\chi}\| \leq \gamma_{0}\right\}$, onde $B_{\gamma_{0}} \in B_{R}$ por (21). Uma vez que $V$ é positiva definida e $\dot{V}$ é negativa definida fora da região $B_{\gamma_{0}}$, conclui-se que $V$ é limitada por um $\bar{V}$ constante e conseqüentemente $\tilde{x}, \tilde{W}_{f}, \tilde{W}_{g i}, \tilde{\psi}$ e $\tilde{\theta}$ são limitados. Em outras palavras, o fato de que $B_{\gamma_{0}}$ é limitada implica que unicamente poderá ocorrer $\dot{V} \geq 0$ para $\tilde{x}, \tilde{W}_{f}$, $\tilde{W}_{g i}, \tilde{\psi}$ e $\tilde{\theta}$ finitos e dentro da região $B_{\gamma_{0}}$, pois em $B_{\gamma_{0}}^{C}$ temos $\dot{V}<0$ e $V$ tem que parar de crescer para valores finitos de $\tilde{x}, \tilde{W}_{f}, \tilde{W}_{g i}, \tilde{\psi}$ e $\tilde{\theta}$, implicando que $\tilde{x}, \tilde{W}_{f}, \tilde{W}_{g i}, \tilde{\psi}$ e $\tilde{\theta}$ são limitados. Objetivando-se provar agora que o erro estendido $\tilde{\chi}$ é ultimamente limitado, escreva (22) como $V=\tilde{\chi}^{T} T \tilde{\chi}$, sendo $T$ uma matriz diagonal, cujos máximo e mínimo autovalores $T_{M}$ e $T_{m}$ são fornecidos pela Hipótese 3. Então, uma vez que $\Omega_{M} \subset \Omega_{m}$, vide comentário 5 , conclui-se que $\tilde{\chi}$, e portanto, $\tilde{x}, \tilde{W}_{f}, \tilde{W}_{g i}, \tilde{\psi}$ e $\tilde{\theta}$ são ultimamente limitados.

Com a finalidade de provar a convergência assintótica do erro de observação, considere os conjuntos disjuntos $\Omega_{x}$ e $\Omega_{x}^{C}$, onde

$$
\Omega_{x}=\left\{\tilde{x} \mid\left\|C^{*} \tilde{x}\right\| \leq z_{0} \exp \left(-z_{1} t\right)\right\}
$$

Adicionalmente, (49) implica

$$
\dot{V} \leq-\alpha_{1}\|\tilde{x}\|^{2}+\psi_{1} \hat{\psi}\left\|C^{*} \tilde{x}\right\|-2 \tilde{x}^{T} P B l
$$

ou, usando-se (8), (24) e (25),

$$
\dot{V} \leq-\alpha_{1}\|\tilde{x}\|^{2}-\frac{\psi_{1} \hat{\psi}\left\|C^{*} \tilde{x}\right\|\left[\left\|C^{*} \tilde{x}\right\|-z_{0} \exp \left(-z_{1} t\right)\right]}{\left\|C^{*} \tilde{x}\right\|+z_{0} \exp \left(-z_{1} t\right)}
$$

Se $\tilde{x} \in \Omega_{x}^{C}$ (ou equivalentemente $\left\|C^{*} \tilde{x}\right\|>z_{0} \exp \left(-z_{1} t\right)$ ), (53) implica

$$
\dot{V} \leq-\alpha_{1}\|\tilde{x}\|^{2}
$$

pois o termo dentro do colchete do lado direito de (53) é maior que zero e $\hat{\psi}(t) \geq 0, \forall t \geq 0$ por (30) e (28).

Por outro lado, se $\tilde{x} \in \Omega_{x}$ a desigualdade (52) implica

$$
\dot{V} \leq-\alpha_{1}\|\tilde{x}\|^{2}+\psi_{1} \alpha_{\hat{\psi}} z_{0} \exp \left(-z_{1} t\right)
$$

onde $\alpha_{\hat{\psi}}=\max \left(\frac{\psi_{1}}{2 \psi_{0}}, \hat{\psi}(0)\right)$.

Com base em (54) e (55) pode ser concluído que

$$
\dot{V} \leq-\alpha_{1}\|\tilde{x}\|^{2}+I^{*} \psi_{1} \alpha_{\hat{\psi}} z_{0} \exp \left(-z_{1} t\right)
$$

onde $I^{*}=\left\{\begin{array}{l}1 \text { se } \tilde{x} \in \Omega_{x} \\ 0 \text { caso contrário }\end{array}\right.$.

Uma vez que $V$ é limitada inferiormente e não crescente para $t \rightarrow \infty$, tem-se

$$
\begin{aligned}
& \lim _{t \rightarrow \infty} \int_{0}^{t}\|\tilde{x}(\tau)\|^{2} d \tau \\
& \quad=\left(V(0)-V_{\infty}\right) / \alpha_{1}+I^{*} \psi_{1} \alpha_{\hat{\psi}} z_{0} / \alpha_{1} z_{1}<\infty
\end{aligned}
$$

onde $\lim _{t \rightarrow \infty} V(t)=V_{\infty}<\infty$. Note ainda que $\|\tilde{x}\|_{\tilde{\tilde{\theta}}}^{2}$ é uniformemente contínua, uma vez que $\tilde{x}, \tilde{W}_{f}, \tilde{W}_{g i}, \tilde{\psi}, \tilde{\theta}$ e $l$ são limitados e, com base em (18), segue que $\dot{\tilde{x}}$ é também limitado. Portanto, aplicando o lema de Barbalat (Ioannou \& Sun, 1996), conclui-se que $\lim _{t \rightarrow \infty} \tilde{x}(t)=0$.

Comentário 6: Note que as condições (30) e (31) podem ser trivialmente satisfeitas, uma vez que as condições iniciais são escolhidas livremente pelo usuário. A condição (32) pode requerer um procedimento de tentativa e erro, pois em (32) são desconhecidos a priori os pesos "ótimos" e limitantes para os erros de aproximação e distúrbios. Contudo, esta deficiência não é peculiar exclusivamente ao esquema proposto, senão à maioria das propostas para identificação baseadas em RNAs. Por exemplo, os esquemas em Polycarpou \& Ioannou, (1991), Kosmatopoulos et alli, (1995), e Yu et alli, (2001), Song, (1998), Jagannathan \& Lewis, (1996), utilizam a projeção de parâmetros, switching- $\sigma$ e zona-morta, que requerem informação prévia sobre o sistema e erro de modelagem.

Comentário 7: Os resultados apresentados nesta seção, baseados em RNAPLs, podem ser modificados para usar RNAs com uma camada escondida (SLHNNs): usando-se a expansão de Taylor com respeito aos pesos para representar as 
componentes de alta ordem da SLHNN (Lewis et alli, 1996), e empregando a metodologia desenvolvida.

\section{SIMULAÇÕES}

Nesta seção são apresentadas simulações com a finalidade de ilustrar os resultados teóricos e a aplicação do esquema proposto. Para tanto, considere o processo descrito por

$$
\begin{gathered}
\dot{x}=A x+B\left(\left[\begin{array}{c}
u \\
-5 x_{2}^{3}+\exp \left(-x_{1}^{2}\right) \cos \left(x_{2}\right)
\end{array}\right]\right. \\
+\left[\begin{array}{c}
0 \\
-0.25 \sin \left(x_{1}^{3}+x_{2}^{2}\right)
\end{array}\right][2+\exp (-t)] \\
\left.+6\left[\begin{array}{c}
\cos \left(x_{2}\right) \cos (2 t) \\
\sin (2 t)
\end{array}\right]\right) \\
y=\left[\begin{array}{ll}
0 & 1
\end{array}\right] x
\end{gathered}
$$

onde $x=\left[\begin{array}{ll}x_{1}, & x_{2}\end{array}\right]^{T}, x(0)=0, A=\left[\begin{array}{rr}0 & 1 \\ 1 & -1\end{array}\right]$, $B=\left[\begin{array}{ll}0.30467 & 0.30467 \\ 0.06152 & 0.06152\end{array}\right]$ e $u$ é um sinal quadrado de amplitude 5 e freqüência 1 . Note que o sistema (58)-(59) é da forma (4)-(5), onde o parâmetro $\theta_{1}=2+\exp (-t)$ satisfaz a Hipótese 2, e por conseguinte, o observador proposto pode ser aplicado.

A primeira fase para implementação do observador (15) consistiu em expressar o termo $C^{*} \tilde{x}$, que aparece no observador e nas leis de adaptação para seus pesos, como função do erro da saída. Para tanto, determinou-se, com base em $C, C^{*}=\left[\begin{array}{ll}0 & 1 \\ 0 & 1\end{array}\right], C^{+}=\left[\begin{array}{ll}0 & 1\end{array}\right]^{T}$ e, na seqüência, $T=\left[\begin{array}{cc}1 & 1\end{array}\right]^{T}$. Portanto, pôde-se concluir que $C^{*} \tilde{x}=$ $\left[\begin{array}{ll}1 & 1\end{array}\right]^{T} \tilde{y}$.

A seguir, foram determinados os valores de $L$ e $P$ que satisfazem simultaneamente as igualdades (7)-(8), isto é a Hipótese 3 , e alocam os autovalores da matriz $Q$ em 6 . Via um processo de tentativa e erro, que incluiu o uso da função ARE do MATLAB, determinou-se $L=\left[\begin{array}{ll}64 & 10\end{array}\right]^{T}$ e $P=\left[\begin{array}{cc}1.03514 & -5.12624 \\ -5.12624 & 41.64014\end{array}\right]$.

As condições iniciais do observador foram escolhidas como $\hat{x}(0)=[-1,-1]^{T}$, para se avaliar o desempenho do observador proposto em condições iniciais desfavoráveis. Os outros parâmetros usados na implementação de (15), (24)-(29) foram

$$
\begin{gathered}
\hat{W}_{f}(0)=0, \quad \hat{W}_{g 1}(0)=0 \hat{\psi}(0)=1, \hat{\theta}(0)=-1, \\
z_{0}=2, \quad z_{1}=0.5, \quad K=I, \quad W_{f 0}=0, \quad W_{g 10}=0, \\
\pi_{f}=\pi_{g 1}=\left[s\left(\hat{x}_{1}\right), \quad s\left(\hat{x}_{2}\right), \quad s(u)\right]^{T}, \\
s(\cdot)=0.1 /[1+\exp -0.1(\cdot)], \\
\alpha_{f}=\alpha_{g i}=\alpha_{\psi}=\alpha_{\theta}=1, \quad C_{f}=C_{g i}=0.1 I, \\
\psi_{0}=0.1, \quad \psi_{1}=10 \text { e } c_{\theta 1}=0.1
\end{gathered}
$$

O desempenho na observação de (58) é apresentado nas Figuras 1 e 2 . Note que as simulações confirmam os resultados teóricos, isto é, o esquema proposto é estável e o erro de observação estado converge assintoticamente para zero.

\section{CONCLUSÕES}

Neste artigo foi proposta uma metodologia para observação adaptativa de sistemas não-lineares incertos com parâmetros variantes no tempo e sujeitos a distúrbios. Foi proposto um observador, baseado em uma parametrização neural e um ganho de realimentação dinâmico, com leis de adaptação para os parâmetros e pesos, que asseguraram a estabilidade do esquema e convergência assintótica do erro residual de observação. Um exemplo de simulação foi considerado para ilustrar a aplicação e desempenho do esquema proposto.

\section{AGRADECIMENTOS}

Os primeiro autor agradece à FAPESP, processos 02/13829-9 e 03/11199-0, e o segundo autor agradece ao CNPq/Pronex, processo 662015/1998-3, pelo suporte financeiro.

\section{REFERÊNCIAS}

Bastin, G. \& Gevers, M. R. (1988). Stable adaptive observers for nonlinear time-varying systems, IEEE Trans. Automatic Control, vol. 33, no. 7, pp. 650-658.

Cho, Y. M. \& Rajamani, R. (1997). A systematic approach to adaptive observer synthesis for nonlinear systems, IEEE Trans. Automatic Control, vol. 42, no. 4, pp. 534537.

Choi, J. Y. \& Farrel, J. A. (2001). Adaptive observer backstepping control using neural networks, IEEE Trans. Neural Networks, vol. 12, no.5, pp. 1103-1112.

Ge, S. S., Hang, C. C., Lee, T. H. \& Zhang, T. (2002) Stable Adaptive Neural Network Control, Kluwer academic publishers, Norwell, Massachusetts, USA. 

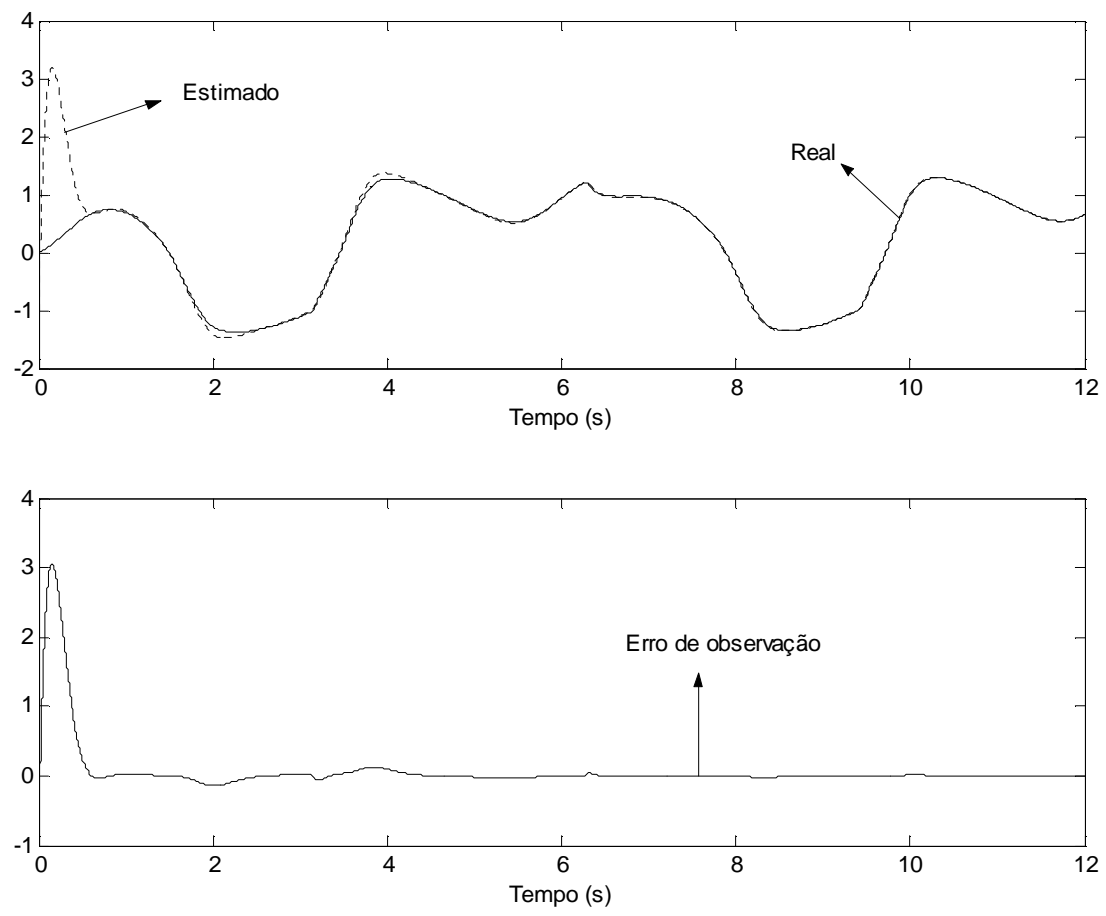

Figura 1: Desempenho na observação de $x_{1}$.
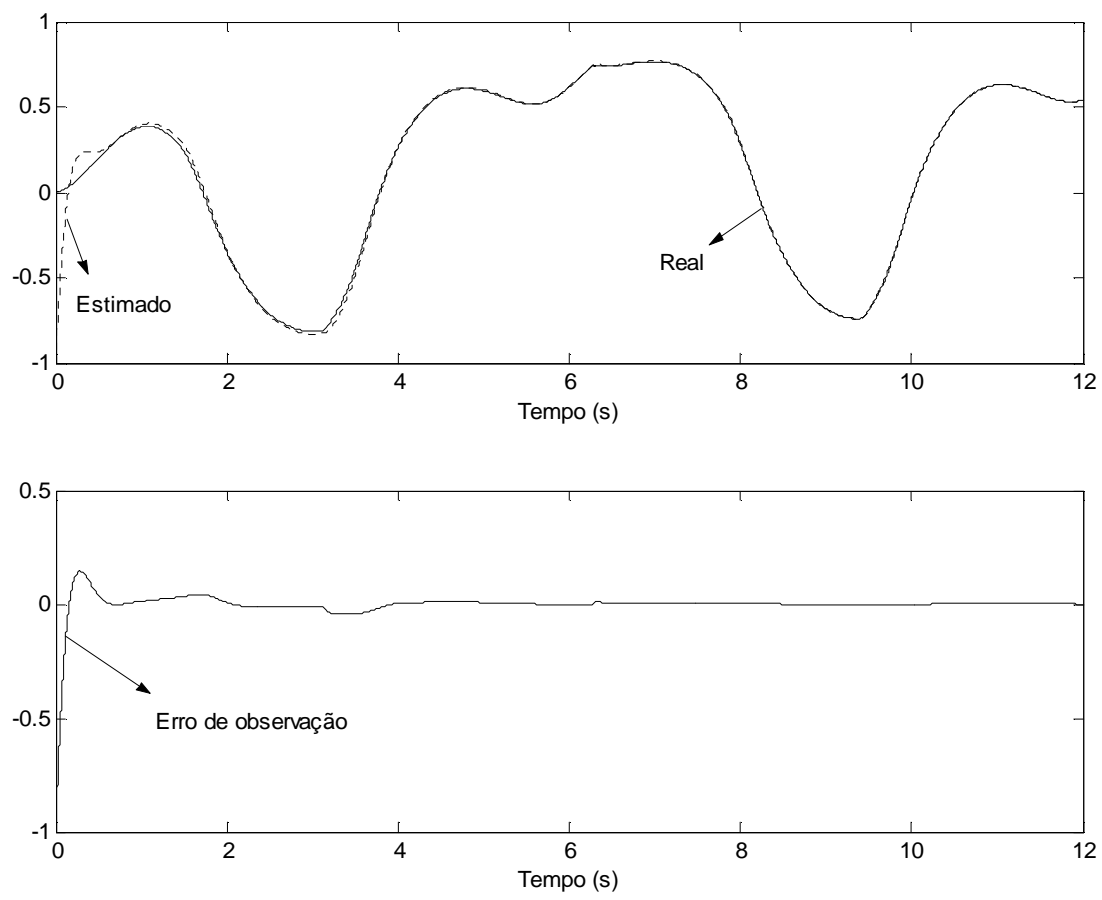

Figura 2: Desempenho na observação de $x_{2}$. 
Ioannou, P.A. \& Sun, J. (1996). Robust Adaptive Control, Prentice-Hall, Inc., Englewood Cliffs, New Jersey, USA.

Jagannathan, S. \& Lewis, F. L. (1996). Identification on nonlinear dynamical systems using multilayered neural networks, Automatica, vol. 32, no. 12, pp. 1707-1712.

Kim, Y. H., Lewis, F. L. \& Abdallah, C. T. (1997). A dynamic recurrent neural-network-based adaptive observer for a class of nonlinear systems, Automatica, vol. 33, no. 8, pp. 1539-1543.

Kosmatopoulos, E. B., Polycarpou, M. M., Christodoulou, M. A. \& Ioannou, P. A. (1995). High-order neural network structures for identification of dynamical systems, IEEE Trans. Neural Networks, vol. 6, no. 2, pp. 442-431.

Lewis, F. L., Yesildirek, A. \& Liu, K. (1996). Multilayer neural-net robot controller with guaranteed tracking performance, IEEE Trans. Neural Networks, vol. 7, no. 2 , pp. 388-399.

Luenberger, D.G. (1966). Observers for multivariable systems, IEEE Trans. Automatic Control, vol. 11, no. 2, p. 190-197.

Marino R. \& Tomei, P. (1995). Nonlinear Control Design: Geometric, Adaptive and Robust, Prentice Hall International (UK) Limited, Redwood Books, Trowbridge, Wiltshire, Great Britain.

Marino, R., Santosuosso, G. L. \& Tomei, P. (2001). Robust adaptive observers for nonlinear systems with bounded disturbances, IEEE Trans. Automatic Control, vol. 46, no. 6, p. 967-972.

Noble, B. \& Daniel, J. W. (1977). Applied Linear Algebra, Prentice-Hall, Inc., Englewood Cliffs, New Jersey.

Polycarpou, M. M. \& Ioannou, P. A. (1991). Identification and control of nonlinear systems using neural network models: design and stability analysis, Tech. Rep. 9109-01, Dept. Elect. Eng. Syst., Univ. Southern Calif., Los Angeles.

Polycarpou, M. M. (1996). Stable adaptive neural control scheme for nonlinear systems, IEEE Trans. Automatic Control, vol. 41, no. 3, pp. 447-451.

Rajamani, R. (1995). Adaptive observers for active automotive suspensions: theory and experiment, IEEE Trans. Control System Technology, vol. 3, no. 1, pp. 86-93.

Rajamani, R. (1998). Observers for Lipschitz nonlinear systems, IEEE Trans. Automatic Control, vol. 43, no. 3, pp. 397-401.
Sanner, R. M. \& Slotine, J. J. (1992). Stable recursive identification using radial basis function networks, Proceedings of the American Control Conference, Chicago, IL, pp. 1829-1833.

Song, Q. (1998). Robust training algorithms of multilayered neural networks for identification of nonlinear dynamical systems, IEE Proc.- Control Theory Appl., vol. 45, no. 1, pp. 41-46.

Vargas, J. A. R. \& Hemerly, E. M. (1999). Robust neural adaptive observer for MIMO nonlinear systems, Proceeding of the 1999 IEEE International Conference on Systems, Man and Cybernetics, pp. 1084-1089.

Vargas, J. A. R. \& Hemerly, E. M. (2005). Algoritmo para identificação assintótica via APLS e sem conhecimento prévio de limitantes, Anais do VII Simpósio Brasileiro de Automação Inteligente, São Luis, Maranhão, Brasil, pp.1-6.

Wang, L. X. (1994). Adaptive Fuzzy System and Control: Design and Stability Analysis, Prentice-Hall, Inc., Englewood Cliffs, New Jersey, USA.

Wang, W. \& Gao, Z. (2003). A comparison study of advanced state observer design techniques, Proceedings of the American Control Conference, Denver, Colorado, USA, pp. 4754-4759.

Yu, W., Poznyak, A. S., \& Li X. (2001). Multilayer dynamical neural networks for non-linear system on-line identification, International Journal of Control, vol. 74, no. 18, pp. 1858-1864.

Zhang, Q. \& Benveniste, A. (1992). Wavelet networks, IEEE Trans. Neural Networks, vol. 3, no. 6, pp. 889-898.

Zhang, Q. (2002). Adaptive observer for multiple-inputmultiple-output (MIMO) linear time-varying systems, IEEE Trans. Automatic Control, vol. 47, no. 3, pp. 525529.

Zhu, R., Chai, T. \& Shao, C. (1997). Robust nonlinear adaptive observer design using dynamical recurrent neural networks, Proceedings of the 1997 American Control Conference, Albuquerque, New Mexico, pp. 10961100 .

Zhu, F. \& Han, Z. (2002). A note on observers for Lipschitz nonlinear systems, IEEE Trans. Automatic Control, vol. 47 , no. 10 , pp. 1751-1754. 